Revue d'histoire de l'Amérique française

RAS REVUE D.HISTOIRE DE L'AMÉRIQUE FRANÇAISE

\title{
Nouveaux besoins, nouvelles cibles? L'histoire rurale et la coopération France-Québec
}

\section{Claire Dolan}

Volume 42, numéro 4, printemps 1989

URI : https://id.erudit.org/iderudit/304740ar

DOI : https://doi.org/10.7202/304740ar

Aller au sommaire du numéro

Éditeur(s)

Institut d'histoire de l'Amérique française

ISSN

0035-2357 (imprimé)

1492-1383 (numérique)

Découvrir la revue

Citer cette note

Dolan, C. (1989). Nouveaux besoins, nouvelles cibles? L'histoire rurale et la coopération France-Québec. Revue d'histoire de l'Amérique française, 42(4), 589-596. https://doi.org/10.7202/304740ar d'utilisation que vous pouvez consulter en ligne.

https://apropos.erudit.org/fr/usagers/politique-dutilisation/ 


\title{
NOUVEAUX BESOINS, NOUVELLES CIBLES? L'HISTOIRE RURALE ET LA COOPÉRATION FRANCE-QUÉBEC ${ }^{1}$
}

\author{
CLAIRE DOLAN \\ Département d'histoire \\ Université Laval
}

Coiffés d'une introduction signée Joseph Goy et Jean-Pierre Wallot, quarante et un textes ont été réunis dans cet ouvrage qui constitue les actes d'un colloque franco-québécois tenu à Rochefort en 1982. Cinq parties divisent le livre: Histoire des comportements démographiques; Économie rurale, économie globale; Famille, mariage, patrimoine et reproduction sociale; Attitudes culturelles et comportements religieux; Histoire de la culture matérielle.

\section{HISTOIRE COMPARATIVE ET ÉCHANGES INTERNATIONAUX}

Le projet de départ était fascinant. Les deux sociétés en cause permettaient de briser le caractère artificiel auquel les histoires comparatives sont trop souvent soumises. La qualité et la réputation des chercheurs qui ont participé au projet témoignent d'ailleurs du sérieux de l'entreprise.

Le programme proposé par les promoteurs du projet partait d'une interrogation ambitieuse et par là même stimulante: «comment les caractéristiques de la société de départ [la société française] se sontelles transplantées dans la société d'arrivée [la société québécoise]?» (p. 14) Le thème conduisait naturellement à une réflexion théorique sur «l'évolution d'un système social: comment la société rurale se fait et se défait, se stabilise, se reproduit ou se transforme» (p. 22).

Il ne faut pas, en ce qui concerne les projets de coopération internationale, juger les résultats à la même aune que celle utilisée pour rendre compte des entreprises individuelles. Plus souvent qu'autrement, les principaux résultats ne se mesurent que partiellement par la

1 À propos du livre Évolution et éclatement du monde rural. France Québec XVIIe-XXe siècles. Structures, fonctionnement et évolution différentielle des sociétés rurales françaises et québécoises XVIIe-XXe siècles. Travaux publiés sous la direction de Joseph Goy et Jean-Pierre Wallot et rassemblés par Rollande Bonnain. Paris/Montréal, Éditions de l'École des hautes études en sciences sociales/Presses de 1'Université de Montréal, 1986. 519 p. 
production scientifique collective qui découle de ces projets. Depuis la tenue du colloque de 1982 dont est issu l'ensemble de textes qui constitue ce livre, plusieurs des participants à la rencontre ont publié d'autres articles et des livres qui dépassent souvent largement leur contribution individuelle à ce colloque. C'est dans ces publications individuelles ou collectives postérieures au congrès de 1982 qu'il faudrait chercher les effets d'une telle rencontre. L'un des drames des organisateurs de colloques est souvent de trouver des éditeurs et des bailleurs de fonds qui ne craindront pas de publier les actes de la rencontre. La publication ne doit pourtant pas trop tarder sous peine de perdre beaucoup de son intérêt. Ici, entre la tenue du colloque, la date de publication des actes et le moment de ce compte rendu, beaucoup trop de temps a passé pour ne pas fausser un peu la portée des résultats.

Le livre n'est pas de peu d'intérêt si on le considère comme un témoin du chemin parcouru par la pratique historienne depuis l'ouverture aux échanges internationaux.

En effet, les échanges de chercheurs entre la France et le Québec se sont développés ces dernières années dans le sens d'une plus grande égalité entre les uns et les autres. Fini le temps où les relations entre les historiens français et québécois prenaient la forme de visites chez nous de «mandarins» qui venaient raconter l'histoire de l'École des Annales à des étudiants et à des professeurs québécois qui l'avaient entendue mille fois. Les échanges aujourd'hui sont sous le signe de la collaboration, d'un apport original de l'une et l'autre partie. Au Québec, comme ailleurs, les générations d'historiens formés depuis les années 1970 ont appris la valeur inestimable de la monographie. On leur a, du même souffle, vanté les mérites de la comparaison. Pas de monographie sérieuse qui ne réfère, pour comparaison, à d'autres lieux, à d'autres temps! Trop souvent cependant, la comparaison se présente comme une formalité visant à moduler le caractère local de la recherche. Les véritables perspectives comparatistes sont rares et leur mise en oeuvre dans des recherches concrètes le sont plus encore. Exercice difficile qui ne semble bien réussir que dans les synthèses qui ferment, le moment venu, les chantiers sur lesquels nombre de tâcherons de l'histoire ont exercé leurs méthodes, usé leur patience et répété ce que leurs maîtres, dans une intuition lumineuse, avaient déjà senti dès l'ouverture du chantier. Le projet dont ce livre est tiré dépassait largement, dans ses buts, le cadre d'une comparaison formelle. Il appelait, dès sa mise en oeuvre, une synthèse, seule capable de rendre toute la richesse des perspectives envisagées. Nul n'aurait osé prétendre que la synthèse souhaitée naîtrait de ces premières rencontres, de ces premiers échanges. Mais nul ne pouvait croire non plus que la synthèse espérée pût naître sans ses échanges. 


\section{ÉVOLUTION DU MONDE RURAL: L'ÉCLATEMENT DE LA RECHERCHE}

On trouvera dans ce livre moins des informations nouvelles sur la société rurale française ou québécoise que la preuve de la vitalité des recherches dans ces domaines, de part et d'autre de l'Atlantique.

Les éditeurs ont pris le parti de conserver au volume la structure du programme du colloque et l'ordre dans lequel les communications y ont été livrées. C'est bien dommage! La structure complètement artificielle qui en découle est parfois irritante et accentue la disparité des thèmes traités.

L'introduction étant une simple présentation des textes, le lecteur doit tirer du recueil ses propres enseignements, puisqu'aucune conclusion ne clôt le volume. Goy et Wallot y placent les objectifs du projet et décrivent l'un après l'autre les apports de chaque contribution en essayant de trouver pour chaque partie un thème intégrateur. Ainsi, les textes de la première partie, "Histoire des comportements démographiques», «soulèvent de grands espoirs en ce qui a trait aux méthodes et résultats de grandes enquêtes en cours» et «font le point sur les acquis ou marquent les limites de nos connaissances actuelles...» (p. 15). La partie II sur l'économie rurale inspire un peu plus les éditeurs qui voient un grand thème la traverser: «la relation plus ou moins forte au marché et son action plus ou moins structurante sur les campagnes.» (p. 16) La partie III est intégrée dans une formule un peu plus précise: «Un système, une société, une couche sociale invente des stratégies plus ou moins conscientes pour perdurer, pour résister aux changements qui menacent son intégrité et pour s'adapter au contraire à ceux qui portent un espoir raisonnable de promotion et de mieux-être.» (p. 18) La partie IV, «Attitudes culturelles et comportements religieux», ne bénéficie que d'une présentation formelle: «La reproduction sociale se double toujours de mécanismes d'encadrement et de reproduction idéologique et culturelle» (p. 20), alors que la cinquième partie sur la culture matérielle est rattachée au reste parce que, "par leurs problématiques, elles [ces recherches] débordent sur l'économie, la reproduction sociale et l'encadrement culturel» (p. 21).

Pourquoi l' «Histoire des comportements démographiques» n'a-telle pas été introduite par l'article de Jean-Pierre Bardet et Hubert Charbonneau qui, tout en ne présentant rien de neuf, avait au moins le mérite de se situer exactement dans la problématique du projet? Les historiens débutants trouveront en tout cas dans ce texte une synthèse utile. Synthèse pertinente également que celle de François Lebrun sur la démographie de la Bretagne. Assez classique, le texte s'appuie sur les travaux récents pour évaluer l'originalité de la Bretagne en termes démographiques. S'il avait été appuyé par d'autres synthèses du même genre sur les autres provinces concernées, l'article aurait été encore plus pertinent. Ouvrant la première partie du recueil, il dispose le lecteur à une 
saisie globale des problèmes démographiques des régions impliquées. Bien disposé, le lecteur aura du mal à se départir d'une certaine irritation, au fur et à mesure qu'il poursuivra sa lecture. Il passera rapidement sur l'article de Claude Renard qui n'apporte rien de neuf, regrettera que le texte de Gabriel Désert n'ait pas été regroupé avec les autres textes qui placent les migrations au coeur de leurs préoccupations, s'interrogera, à la lecture de l'article méthodologique essentiel de Gérard Bouchard sur la définition que les éditeurs ont donnée aux «comportements démographiques» et se demandera comment les autres auteurs du recueil ont pu, sans même se justifier, tomber si facilement dans les pièges que Bouchard met à jour² .

Les 170 pages de la deuxième partie réunissent des textes qui traitent de l'économie rurale. Enlevons d'entrée de jeu l'article de John A. Dickinson qui compare la conception populaire de la tenure en Normandie et en Nouvelle-France, dont l'intérêt pour le projet est incontestable et qu'on aurait été mieux inspiré de placer avec les attitudes culturelles. Le texte de Laurier Turgeon subira la même exclusion: pêcheurs basques et indiens du XVIe siècle n'ont rien à faire dans l'économie rurale. Épurée de ces appendices, la deuxième partie ne présente rien de comparable aux synthèses de la première partie: la comparaison entre les deux sociétés est ici complètement factice ou simplement mise de côté. Le choix de Micheline Baulant de comparer trois fermes céréalières françaises en pays boisé pour «offrir à la comparaison francocanadienne un terroir conquis sur la forêt, même si l'époque des défrichements remontait à plusieurs siècles» (p. 107) manifeste la volonté louable de s'inscrire dans un cheminement comparatif sans pour autant éviter les dangers de la caricature. Même volonté louable dans la présentation alternée des zones marginales rurales au Québec et en France par Normand Séguin et Françoise-Eugénie Petit. Plutôt que dans la comparaison entre les deux économies, l'intérêt de l'article réside dans la largeur des perspectives des auteurs qui échappent au caractère local de leurs recherches pour toucher des problèmes beaucoup plus universels. Les autres textes de cette partie sont pour la France des monographies précises, sans lien les unes avec les autres, micro-histoire qui évite de remettre l'étude locale en perspective, sauf pour Jean-Pierre Chaline qui utilise, pour mettre en cause la domination de la ville sur la campagne, la comparaison avec le reste de la France. Les textes québécois confirment quant à eux l'impression qu'un dialogue de sourds s'est installé une fois pour toutes entre les historiens qui traitent de l'économie rurale québécoise et que bien malin ou bien habile sera celui qui réussira à en tirer une quelconque synthèse. Corinne Beutler, Christian Dessureault et Mario Lalancette optent pour des études de cas. Leurs textes ne prétendent pas définir la conjoncture économique géné-

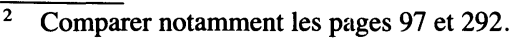


rale ni les transformations économiques de la société rurale québécoise. Par contre, l'article de Jean-Pierre Wallot et Gilles Paquet, celui de Louise Dechêne et la première partie du texte de Normand Séguin posent chacun de façon différente les mêmes problèmes. Tout dans ces textes quand on les place côte à côte appelle un débat. Le parti pris de WallotPaquet d' «une modernisation de la socio-économie québécoise dans chacun de ses segments géographiques importants au tournant du XIXe siècle» (p. 239) est soutenu par le fait que les auteurs assurent qu'il y a eu «accroissement des niveaux de richesse et modernisation des structures sociales (p. 239). On reste étonné devant l'ambition de la proposition mais complètement interloqué par la source qui permet aux auteurs de telles affirmations: l'inventaire après décès. Le problème reste entier, à la fin du texte: on est loin d'être convaincu que la source est utilisable pour les fins auxquelles on la fait servir, et on ne sait toujours pas ce que les auteurs entendent par la modernisation de la socio-économie québécoise. Louise Dechêne place son texte dans la suite du débat sur l'agriculture au XIXe siècle et elle insiste sur les disparités régionales qui entraînent des «efforts d'ajustement» différents, d'une région à l'autre (p. 190). Normand Séguin prend prétexte du développement de la marginalité rurale pour remettre en cause les interprétations trop rapides: dans le «processus d'intégration de la production agricole à l'économie de marché», une grande "variété de situations» existe. Polymorphisme qui se résout plus par l'identification des dominantes d'une époque et d'une autre que par un passage définitif d'une économie d'autoconsommation à une économie de marché (p. 262). Le lecteur peu au fait des débats historiques autour de l'économie agricole québécoise au XIXe siècle retiendra de cette partie l'impression que la chronologie des changements est floue, peu rigoureuse; peut-être serat-il intrigué par les contradictions qu'il décèlera entre les interprétations; il aurait alors beaucoup de mérite car rien dans l'introduction ne lui annonçait quelque divergence que ce soit. Un débat entre les auteurs serait ici beaucoup plus sain que leur cheminement en parallèle dans l'histoire de l'économie rurale. Dans la poursuite des travaux sur la société rurale, il ne faudrait pas l'exclure. Le lecteur et l'histoire gagnent toujours à ce que soient discutées les différentes interprétations, la question pourrait peut-être de ce fait être sinon éclaircie du moins clarifiée. On mesure ici tout l'intérêt d'une véritable comparaison avec la France.

Dans la troisième partie, «Famille, mariage, patrimoine et reproduction sociale», apparaît avec le plus d'acuité le résultat d'une séparation artificielle entre les anciennes catégories historiques (démographie) et les nouvelles (famille): l'article de Jean Tarrade sur l'exogamie et l'endogamie qui confirme ce que l'on sait déjà aurait avantageusement pu être rapproché de celui de Gabriel Désert sur les migrations; un peu artificiel aussi paraîtra le raccrochement de l'article de René Plessix au thème de la famille parce qu'il utilise les tables de contrats 
de mariage pour déterminer les catégories sociales. Puisque leur objet n'est pas si éloigné, cet article et celui de Gérard Bouchard se seraient très bien prêtés à un regroupement. L'article de Jean-Paul Desaive est aussi un article méthodologique. Grâce au couplage des données généalogiques et du centième denier, il évalue les modifications de la propriété paysanne à partir des partages, des échanges, des achats et des ventes et conclut que la plupart des contractants ne font que quelques transactions, les héritages constituant la principale façon d'accroître la propriété foncière (p. 302). Jacques Mathieu et son équipe auxquels Tiphaine Barthelemy de Saizieu peut être associée étudient la mobilité au mariage et la cohésion sociale. Ils établissent entre autres que «le recours au conjoint étranger devient un moyen de resserrer la cohésion sociale» dans une «communauté rurale peuplée depuis au moins deux générations» (p. 306). À partir de l'exemple de Neuville, c'est un modèle qui s'élabore et qui remet en cause les clichés trop souvent véhiculés sur les migrations et leurs causes. Ici la spécificité de la Nouvelle-France est claire, elle contribue à montrer l'intérêt de traiter la mobilité au mariage autrement qu'à travers des catégories surannées qui répètent des évidences maintenant bien connues. Peut-être les travaux en cours de Louis Michel et Louis Lavallée apporteront-ils le même renouvellement. Ce qu'ils annoncent est ambitieux mais les textes qu'ils présentent ici ne permettent pas de dépasser les hypothèses ou les généralités déjà bien connues. Cette section se termine par un article de Louis Assier-Andrieu qui traite de Léon Gérin, disciple de Frédéric Le Play. L'idée d'introduire ce texte est intéressante. Dommage que, dans le contexte, sa pertinence ne soit pas évidente. En effet, personne parmi les participants ne s'est référé à Le Play et un seul ${ }^{3}$ a cité Gérin. La présence de cet article dans le recueil met en évidence la faiblesse théorique de la plupart des textes du recueil. Pouvait-on cependant demander que des textes si courts aillent au-delà?

Malgré son titre, il est assez difficile de résumer en quelques mots l'apport de cette partie. En fait, on y apprend peu de choses sur la famille, beaucoup plus sur le patrimoine, et la reproduction sociale ne s'y présente malheureusement que comme une hypothèse ou un prétexte à exercices méthodologiques. C'est là d'ailleurs un des paradoxes de ce recueil: il porte, à certains moments, la réflexion méthodologique à un très haut niveau (p. 89-103); mais il se contente, dans d'autres contributions, d'expliquer inutilement des techniques élémentaires bien connues (p. 326-327).

Des attitudes culturelles et des comportements religieux qui constituent la quatrième partie de l'ouvrage, je ne retiendrai que les articles de Catherine Velay-Vallantin, René Hardy et Jean Roy, Charles

3 Luce Vermette, «Les donations: activités domestiques et genre de vie 1800-1820 et 1850$1870 », 513$, note 8 . 
Frostin et André Lachance qui présentent un intérêt. Celui de Vallantin parce qu'il propose d'aborder le transfert d'attitudes culturelles françaises au Québec par l'intermédiaire de la diffusion des contes oraux et écrits. Des trois exemples présentés par l'auteure, il ressort que la «rencontre entre des réponses fantasmatiques à des situations nouvelles et les représentations de réalités socio-historiques» (p. 395) pourra apporter à l'étude des attitudes culturelles un éclairage nouveau. À la condition toutefois que l'étude du conte ne s'écarte pas trop des réalités sociohistoriques qu'il est censé représenter. Charles Frostin aborde la «mission» canadienne à partir du contexte français qui la soutient et remet en perspective le contexte qui la produit. Article fort révélateur que plusieurs rateront parce qu'il n'est vraiment pas à sa place ici: bien loin du monde rural, il n'a rien à voir non plus avec les attitudes culturelles de celui-ci. Et pourtant, il mérite qu'on souligne son intérêt pour tous ceux qui travaillent sur le XVIIe siècle québécois, pour tous ceux qui sont aux prises avec les Relations des Jésuites. René Hardy et Jean Roy présentent, à partir du contenu des rapports annuels des curés, une étude des «effets des enseignements du clergé sur les mutations de la culture religieuse» (p. 397). Ils montrent entre autres que si le début de ce siècle est caractérisé par «l'unanimité de la pratique pascale et [...] une plus stricte observance des prescriptions de l'Église», cela tient en grande partie à la mise en place d'institutions d'encadrement qui succèdent à une période où la dissidence demeure passablement importante (18501875) (p. 407). André Lachance utilise une notion de délinquance qui me semble beaucoup trop large. Considérer les chicanes entre voisins comme des comportements délinquants me semble en effet un peu sévère. Les textes de Jacques Marcadé et Roger Dupuy, centrés sur leur objet spécifique, s'intègrent difficilement à l'ensemble. L'article de Yvonne Pasquet sur l'alphabétisation aurait pu amorcer un échange, même si on n'y apprend rien de neuf, mais le recueil ne contient rien sur les recherches qui s'effectuent au Québec sur la question.

D'intérêt très inégal, cette partie confirme que l'appellation «attitudes culturelles» permet d'intégrer à peu près tout. Relativement peu traité à l'intérieur du recueil, le champ me paraît un des plus prometteurs toutefois et il faudrait bien un jour établir un véritable programme de recherche comparée pour élucider systématiquement des problèmes qui risquent de bénéficier le plus de la comparaison avec la France.

Si les attitudes culturelles ont été négligées, que dire de l'appendice que constitue la cinquième partie, l'«Histoire de la culture matérielle»? Essentiellement descriptive, cette partie n'apporte rien à la compréhension du monde rural, ni français ni québécois et elle reste au niveau des «curiosités». Soulignons toutefois la contribution de Nicole Pellegrin qui utilise les inventaires après décès avec finesse et présente un projet sur l'histoire de l'habillement rural qui réconcilie le lecteur à la fois avec les inventaires après décès et avec la culture matérielle en général. 
Plusieurs tableaux complètent les textes mais on n'en trouve la liste nulle part, ce qui est peut-être un peu dommage.

\section{L'AVENIR DE LA PRATIQUE HISTORIENNE ET LES COLLABORATIONS INTERNATIONALES}

J'ai annoncé d'entrée qu'on ne pouvait s'attendre à trouver ici les réponses aux questions formulées par les initiateurs du projet. La décision des organisateurs de publier tous les textes du colloque (sauf un) nous permet de constater l'importance du projet et l'intérêt qu'il suscite chez les chercheurs de part et d'autre. Elle nous indique aussi que l'entreprise n'est pas facile à mener à son terme, que les critères scientifiques de conduite d'une étude ne sont pas toujours compatibles avec les critères politiques qui président parfois au destin des projets de coopération internationale. Scientifiquement, on souhaiterait que les meilleurs chercheurs s'attèlent à la tâche, secteur par secteur, en privilégiant un problème à la fois, et ce, des deux côtés de l'Atlantique. Les collaborations devraient entre autres tenir compte des effets d'une chronologie trop lâche qui dilue ici les aspects comparables. Le titre du recueil parlait d'évolution, il faut bien se garder de prendre pour une évolution le simple fait que l'on place des points à plusieurs endroits sur la ligne du temps. On souhaiterait par ailleurs que les échanges puissent s'inscrire dans un plus long terme, afin de permettre aux chercheurs non pas seulement de faire connaissance mais de vraiment apprendre à travailler ensemble. On souhaiterait aussi que la communauté scientifique puisse disposer le plus tôt possible des textes terminant chacune des étapes.

Il faut voir ce livre non pas comme la présentation des résultats d'un projet terminé mais plutôt comme autant de chemins tracés pour de nouvelles collaborations: les chercheurs ont repéré leurs intérêts communs, les voies sont ouvertes pour que plusieurs sous-projets d'histoire comparative découlant de ces premières expériences aient une chance de réussir. Les deux communautés scientifiques impliquées ne peuvent que souhaiter que de telles initiatives se poursuivent, malgré les lacunes, malgré les tâtonnements qu'elles supposent. L'étape de la mise sur la table des recherches individuelles a été franchie. Espérons qu'après un tri sévère mais nécessaire, on fixera à la collaboration des objectifs plus restreints et que l'on continuera, dans le cadre d'étapes bien définies, à chercher réponse à l'interrogation de départ. Lourd défi auquel il faut consacrer beaucoup de temps mais l'histoire y gagnera peut-être la meilleure justification de la comparaison véritable, seul moyen pour arrêter le processus d'accumulation de monographies parallèles dans lequel la pratique historienne a tendance à s'installer. 